\title{
Entwicklung eines integrierten optoelektronischen Berührungssensors
}

\author{
G. Kodl ${ }^{1}$, C. Voigt ${ }^{2}$, J. Tremmel ${ }^{2}$, and J. Siegl ${ }^{2}$ \\ ${ }^{1}$ Leoni AG, Zentrale Forschung und Entwicklung, Nürnberg, Germany \\ ${ }^{2}$ Georg-Simon-Ohm Fachhochschule Nürnberg, Germany
}

Zusammenfassung. Für die von der Firma LEONI AG, Nürnberg entwickelte "dämpfungssensitive" flexible Polymerfaser ergeben sich vielfältige Anwendungen, u.a. im Automotive-Bereich, beispielsweise als Einklemmschutzsensor oder als Fußgängerschutzsensor integriert in die Stoßstange. Auf der Sendeseite wird in die Plastikfaser ein gepulstes Lichtsignal eingespeist. Am Empfangsort ist der Photostrom einer Photodiode abhängig vom Druck der auf die Faser einwirkt. Die integrierte Sensorelektronik hat die Aufgabe, den vom Druck beeinflussten Photostrom zu erfassen und möglichst störsicher auszuwerten. Die Anforderungen verschiedener Applikationen müssen sich durch geeignete Konfiguration der Auswertesoftware berücksichtigen lassen. Die integrierte Sensorelektronik besteht aus einem Licht/Frequenz-Konverter und einer auf Basis eines Prozessors implementierten Auswertesoftware. Der Licht/Frequenz-Konverter ist ein getakteter, konfigurierbarer Integrator, der über einen Komparator ein Digitalsignal liefert, dessen Frequenz proportional dem Photostrom ist. Sensorelektronik und Photodiode wurden in CMOS-Technologie mit dem 0,35um-CMOS-Opto-Process der Firma Austria Microsystems, Graz, realisiert.

\section{Systemkonzept}

Den Systemaufbau des optoelektronischen Berührungssensors zeigt Abb. 1. Eine von einem Microcontroller angesteuerte optoelektronische Sendediode speist Lichtleistung in die berührungssensitive Polymerfaser. Die Sensorfaser ist dadurch gekennzeichnet, dass sie druckabhängig die Dämpfung verändert. Damit ist der Photostrom der Empfängerdiode druckabhängig bzw. berührungssensitiv. Der optoelektronische Wandler im Empfänger liefert am Ausgang ein digitalisiertes Signal, das vom Microcontroller ausgewertet wird.

Correspondence to: J. Siegl (johann.siegl@fh-nuernberg.de)

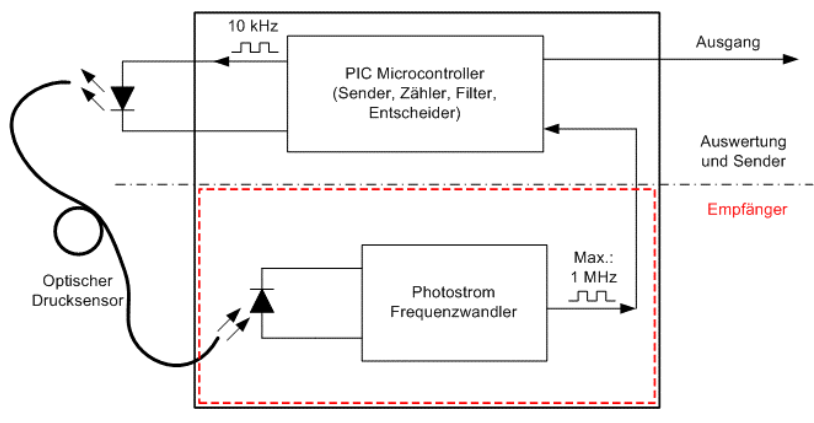

Abbildung 1. Systemaufbau des optoelektronischen Berührungssensors.

Das ausgewertete Ergebnis über die Größe des einwirkenden Druckes wird schließlich über eine Standard-Schnittstelle einer übergeordneten Systemeinheit übermittelt. Ziel ist es, die gesamte Sensorelektronik einschließlich der Photodiode und Auswerteeinheit kompakt in CMOS-Technologie zu integrieren. Dabei geht es u.a. um die Beherrschung von Drifteffekten, der Eliminierung des Dunkelstroms und der Unterdrückung von Temperatureinflüssen.

\section{Sensorelektronik}

Die wichtigste Komponente der Sensorelektronik ist die Photostrommessung und Digitalisierung. Am zweckmäßigsten ist dabei ein integrierendes Wandlerprinzip, bei dem der Photostrom eine Kapazität auflädt bzw. entlädt. Ein speziell ausgelegter Komparator erzeugt gemäß seiner Ansprechschwellen ein digitalisiertes Signal am Ausgang (siehe Abb. 2). In einer ersten Testschaltung wurden verschiedene Varianten für die Photostrom/Frequenzwandlung unter Nutzung des Design-Kit (HIT-KIT) der Firma Austria Microsystems entwickelt. Dieses Design-Kit stellt analoge und digitale Schalt-

Published by Copernicus GmbH on behalf of the URSI Landesausschuss in der Bundesrepublik Deutschland e.V. 


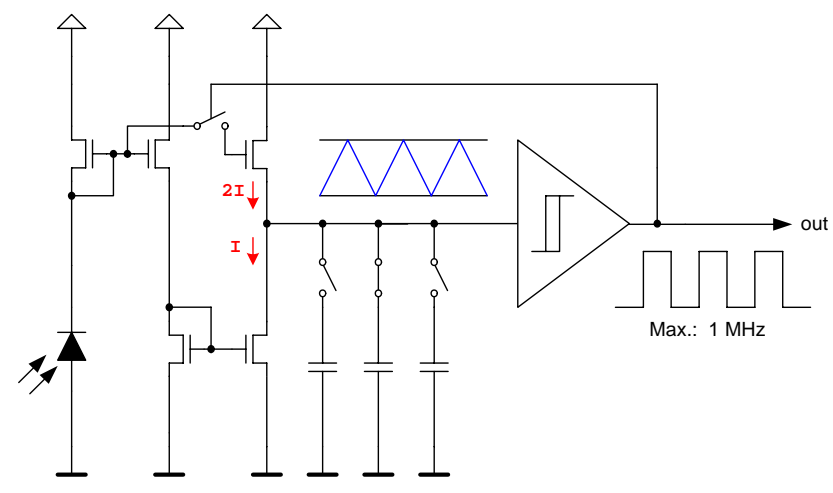

Abbildung 2. Prinzipschaltbild der Photostrommessung mit konfigurierbarer Integrations-Kapazität (integrierendes Wandlerprinzip) mit Komparator und frequenzproportionalem Digitalausgang.

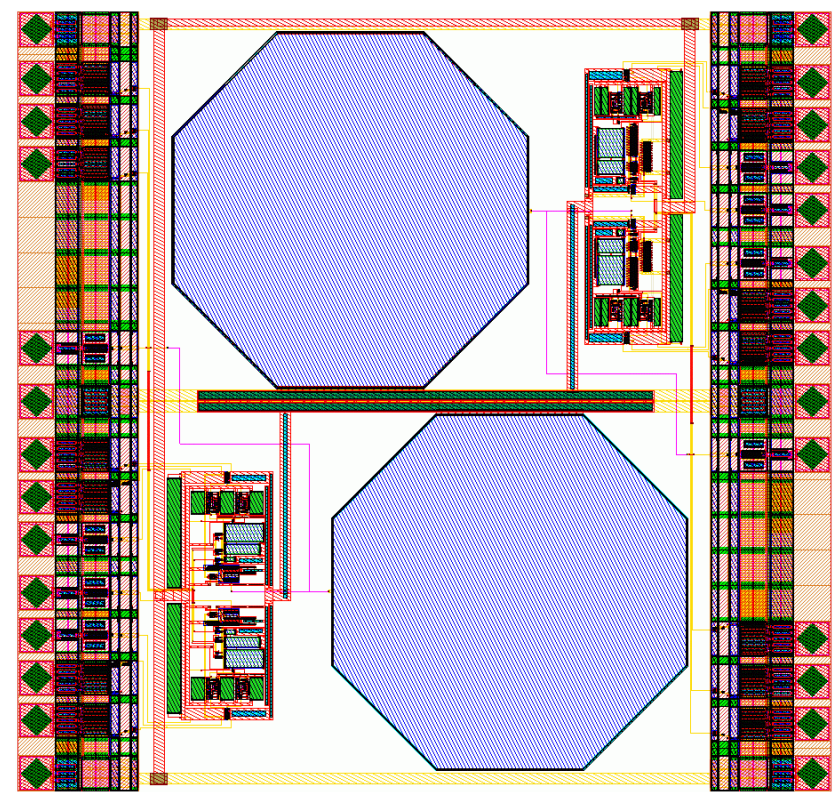

Abbildung 3. IC-Layout von verschiedenen Realiserungsvarianten einschließlich Photodiode für den Photostrom/Frequenzwandler erstellt mit der IC-Station der Firma Mentor Graphics.

kreiszellen für den 0,35um-CMOS-Process zur Verfügung. An vorentwickelten analogen Zellen können u.a. ausgewählte OP-Verstärker und Komparatoren verwendet werden. Allerdings war es erforderlich, u.a. einen eigenen Komparator zu entwickeln, wegen der besonderen Anforderungen betreffs der Geschwindigkeit, der Stabilität der Ansprechschwellen und der "Stromergiebigkeit" am Ausgang. Im Weiteren erfolgte die Untersuchung geeigneter Wandlerkonzepte u.a. basierend auf dem Prinzip der sukzessiven Approximation. Ein D/A-Wandler gibt dabei "Stromgewichte" vor, die in einem Strom-Komparator verglichen werden und je nach Vergleichsergebnis das Approximationsregister setzen.

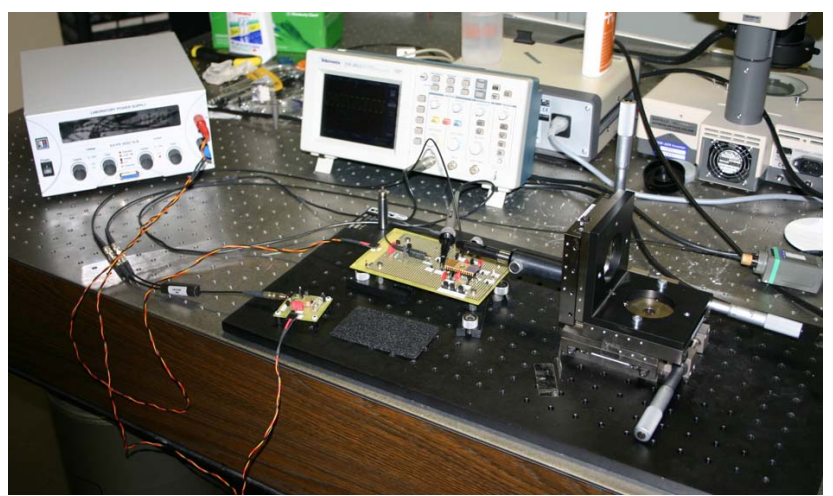

Abbildung 4. Messaufbau für den Test der Entwicklungsmuster.

\section{IC-Design}

In Abb. 3 ist das Chip-Layout der realisierten Schaltungsvarianten dargestellt. Deutlich erkennbar sind wegen der großen Fläche die achteckigen Photodioden. Daneben befindet sich die Photostrom/Frequenzwandler jeweils in zwei Realisierungsvarianten, die als A/D-Wandler wirken. Das Ausgangssignal wird im Weiteren von einem Processor ausgewertet und verarbeitet. In einer nächsten Entwicklungsstufe soll die Photodiode, die A/D-Wandlerschaltung und der Processor auf einem Chip integriert werden. In einer Vorarbeit wurde ein synthesefähiges VHDL-Modell eines 8-Bit-Processors entwickelt und mit der Auswertesoftware getestet. Für den Entwicklungsprozess wurden Designwerkzeuge der Firma Mentor Graphics, u.a. DesignArchitect, Eldo, SystemVision und die IC-Station verwendet. Für das Testdesign wurden im Rahmen des Europractice-Programms Entwicklungsmuster vom Halbleiterhersteller realisiert und geliefert.

\section{Auswertesoftware}

Die Auswertesoftware auf Basis eines 8-Bit-Microcontrollers wurde möglichst ressourcenschonend ohne Betriebssystem realisiert. Durch geeignete Filterung und Differenzbildung bei der Messwerterfassung ist es möglich, Drifteinflüsse zu kompensieren. Im Weiteren kann durch bereichsweise definierbare Schwellenwerte das Auslöseverhalten auf den jeweiligen Anwendungsfall angepasst werden.

\section{Messergebnisse}

Zunächst wurden bei den gelieferten ICs die DCSpannungen und -Ströme überprüft, sowie die Ruheströme (Photodioden abgedeckt) und die Ströme bei Beleuchtung der Photodioden. Die Messergebnisse der DC-Analyse stimmen mit den Werten der Simulation überein, der Biasstrom der Komparatoren beträgt wie gewünscht und simuliert in etwa $20 \mu \mathrm{A}$. Anschließend wurde u.a. die Abhängigkeit der 


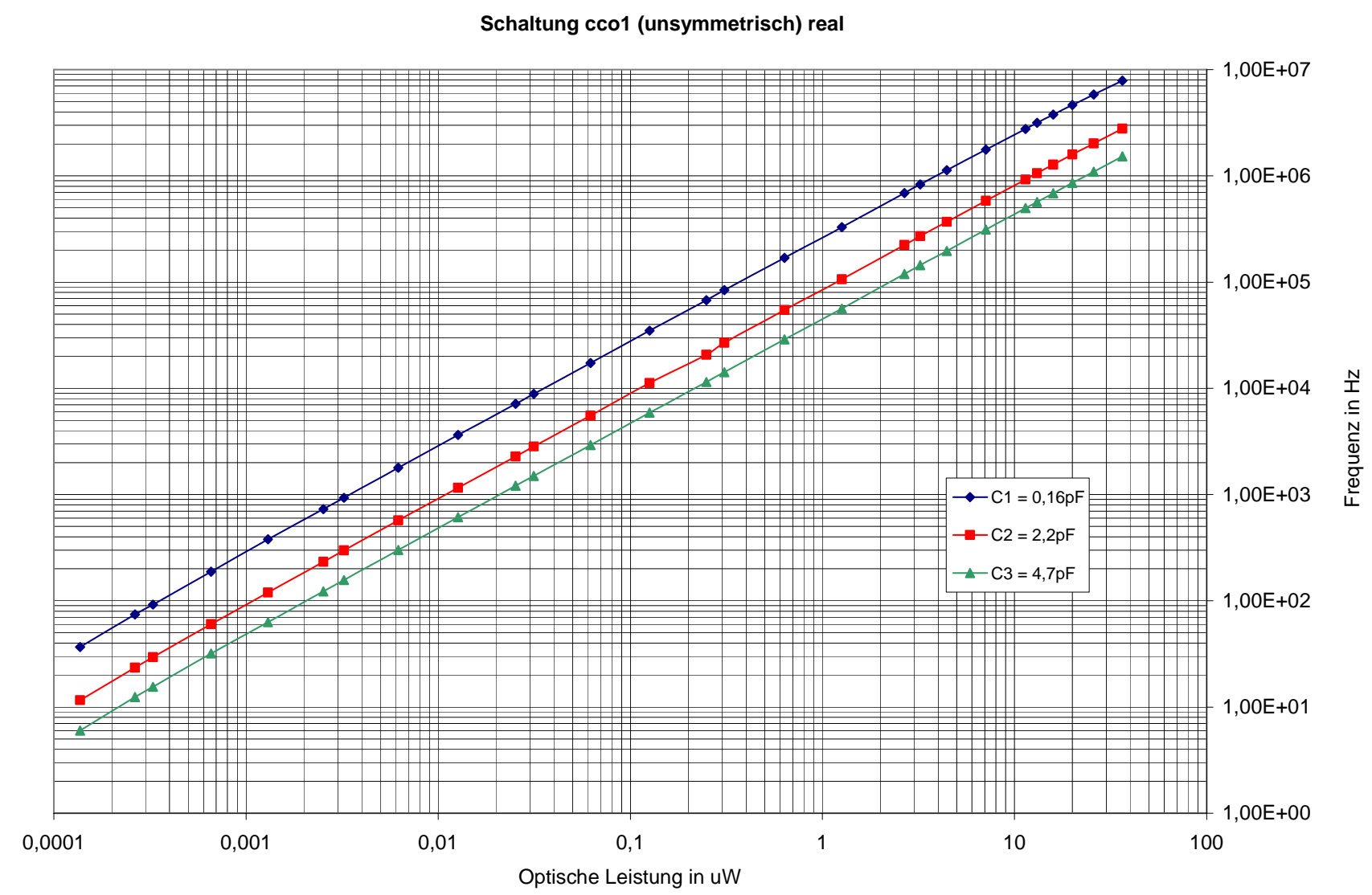

Abbildung 5. Abhängigkeit der Ausgangsfrequenz von der Lichtleistung (Variante 1).

Ausgangsfrequenz der Schaltung von der auf die Photodiode treffenden Lichtleistung ermittelt. Den Messaufbau für den Test der Entwicklungsmuster zeigt Abb. 4.

Vor Beginn der Messungen wurde die Faser mit Hilfe der x,y,z Positionierhilfe so ausgerichtet, dass sich die maximale Ausgangsfrequenz am Ausgang der jeweiligen Schaltung einstellt (Schaltungsvarianten: u.a. Variante cco1 - Kapazitäten werden unsymmetrisch geladen/entladen und Variante cco2 - Kapazitäten werden symmetrisch geladen/entladen). Nach Konfiguration und damit Festlegung verschiedener Kapazitäten in der Empfängerschaltung erfolgte dann die Einstellung einer definierten Lichtleistung in der Sendeschaltung. Für die auf die integrierte Photodiode treffende unterschiedliche optische Lichtleistung wurden schließlich die entsprechenden Ausgangsfrequenzen der Wandlerschaltung ermittelt. Abb. 5 zeigt die ermittelten Abhängigkeiten der Variante 1.

\section{Zusammenfassung}

In einem Testaufbau wurden die gelieferten Entwicklungsmuster eingehend in einem Temperaturbereich von $-40^{\circ} \mathrm{C}$ bis $+120^{\circ} \mathrm{C}$ getestet. Die Simulationsergebnisse konnten dabei bestätigt werden. Die gestellten Anforderungen werden voll erfüllt.

\section{Literatur}

Patente: DE 19721341 C2, DE 3603934 A1, DE 3919743 C2, GB 2201511 A1, GB 2288014 A,

Patente: EP 0066 493, DE 4236 742, DE 19726 731, DE 3802 527, DE 39 19 743, DE 3628 715, DE 3912141 